\title{
Competitive Failure of Bolt Loosening and Fatigue under Different Preloads
}

\author{
Guangwu Yang, Long Yang* ${ }^{*}$, Jingsong Chen, Shoune Xiao and Shilin Jiang
}

\begin{abstract}
Existing research on the competitive failure relationship, failure mechanism, and influencing factors of bolt loosening and fatigue under different preloads is insufficient. This study analyzes the competitive failure relationship between bolt loosening and fatigue under composite excitation through competitive failure tests of bolt loosening and fatigue under different preloads. The results indicated that the failure mode of the bolt is only related to the load ratio $(R)$ and is unrelated to the initial preload and excitation amplitude, which only determine the failure life of the bolt. The small axial loads of composite excitation can restrain bolt failure, and the significant degree of this restraining effect is different for different preloads. Subsequently, a fracture analysis of the bolt was performed to verify the competitive failure relationship of the bolt from a microscopic perspective, and the competitive failure mechanism of the bolt was determined. Based on the findings, we propose a calculation equation for the optimal preload of 8.8 grade high-strength bolts that can serve as a reference for engineering applications.
\end{abstract}

Keywords: Bolt, Preload, Loosening, Fatigue, Competitive failure

\section{Introduction}

A bolt is a standardized part that is widely used in rail vehicles, aircraft, chemical machinery, automobiles, ships, and other industrial equipment owing to its simple structure, easy disassembly, reliability, and numerous other advantages. The main function of a bolt is to reliably connect two or more components. In pressure vessels and related equipment, bolts are combined with sealing washers to improve the effectiveness of the seal, which requires the application of preloads on the bolted connection [1]. In complex working environments, bolted connections can be subjected to impact, vibrations, thermal stress cycles, and other loads. This can gradually reduce the preload, and the bolt can become loose. Although the normal operation of the system may not be affected during the initial stages of loosening, as the preload continues to decrease, the loosening intensifies, further reducing the overall stiffness of the entire bolted

*Correspondence: yanglong6788@163.com

State Key Laboratory of Traction Power, Southwest Jiaotong University, Chengdu 610031, China connection system and resulting in bolt fracture. In addition, when bolted connections are subjected to large axial loads, direct fatigue fracture can also occur without loosening.

The primary factors that affect bolt loosening are the initial bolt preload, action mode of the working load, parameters of the bolt structure, working environment temperature, material of the clamped parts, and contact state [2]. The primary factors that affect bolt fatigue include the bolt initial preload, external load, bolt material, processing method, heat treatment procedure, etc. Thus, the preload is an important factor that affects bolt loosening and fatigue failure. Considering external loads, transverse loads perpendicular to the screw direction are the primary factor of bolt loosening [3], whereas axial loads along the screw direction are the primary factor of bolt fatigue [4]. Furthermore, as the service time of the bolted connection structure increases, the preload decreases to a certain extent, which can lead to bolt loosening and fatigue. In extreme cases, it can also cause major accidents such as bolt fracture and structural 
disintegration. Therefore, bolt loosening and fatigue failure under different preloads must be studied in detail.

The preload exerted on a bolt during the tightening process directly determines the clamping force between the two clamped parts. Insufficient preloading can lead to bolt loosening, whereas excessive preloading can lead to bolt overload fractures. Therefore, while designing bolted joints, the required tightening torque must be accurately analyzed, and the appropriate preload must be precisely determined to prevent loosening and enhance the reliability of bolted connections. The following preload application methods are generally used during a bolt assembly process [5]:

(1) Torque control method

(2) Turn-of-the-nut method

(3) Nut rotating method

(4) Stretching method

(5) Bolt tightening method

The torque control method is the most widely used method for engineering applications. The calculated torque value is often based on the yield point, such that the bolt preload is close to the yield strength of the bolt material, but does not exceed $80 \%$ of the material yield limit considering the safety factor. The yield strengths of bolts vary based on the material of the bolt, and consequently, the maximum preload varies as well. During the bolt tightening process, the clamping force between the clamped parts increases rapidly with the increase in the bolt preload. When the bolt reaches the yield point, it undergoes plastic deformation. At this stage, the clamping force increases negligibly or remains unchanged. If the preload continues to increase further, the clamping force gradually decreases until the bolt fractures [6]. Under high preloads and alternating loads, high-strength bolts often experience fatigue fracture [7]. Consequently, the loosening life and fatigue strength of bolts must be investigated considering different preloads.

Several studies have been conducted to determine the influence of preloads on bolt loosening. The studies are mainly manifested in the loosening process, simulation method and influence law. Experts and scholars have made a lot of contributions in the study of the dividing method of loosening process. For example, Wang et al. [8] divided the bolt loosening process into two stages. During the early loosening stage, the initial preload gradually relaxes due to the periodic plastic deformation of the material, but the nut does not rotate; the second loosening stage is characterized by the obvious recession of the nut and the rapid recession of the clamping force. Furthermore, Jiang et al. [9] revealed that a nut rotation angle of $0.5^{\circ}$ acts as the boundary between the two stages. Although many dividing methods are proposed, there is no explicit and unified method for the dividing of each stage of the recession curve of the bolt clamping force. In terms of the finite element simulation of bolt loosening, Ke [10] and Wang [11] proposed a finite element simulation analysis method to study the loosening of bolted connection structures by applying preloads using the rotation angle method. The proposed method can be used to carry out multi-stage and whole-process finite element simulation analyses of the loosening of bolted connection structures under transverse vibrations. Lu [12] established a three-dimensional solid finite element model of a bolted connection structure considering different preloads and contacts and calculated the stress distribution of the bolts under different preloads. The results revealed that the structural loosening characteristic decreases with the increase in the bolt preload. Gong et al. [13] proposed a simulation method to quantitatively determine the critical load of transverse loosening. He determined that the anti-loosening ability of a bolted connection can be improved by increasing the preload and friction coefficient and reducing the clamping length and fit tolerance. It can be seen that the simulation of bolt loosening and the application of preload are relatively mature enough to meet the needs of engineering design or verification. In the study of the influence of preload on bolt loosening, Zheng et al. [14] studied the loosening response of threaded connections under a series of impact loads using the Hopkinson bar test and established a relationship between the change in the preload and the loosening swing angle of the nut. Sanclemente et al. [15] performed bolt loosening tests under transverse loads and found that the preload and elasticity of the fasteners are the main factors that affect bolt loosening. Furthermore, he established a statistical model to predict the degree of bolt loosening, and concluded that a high preload and low elastic modulus can reduce the rate of loosening. Zhang [16] analyzed the influences of the preload, lateral amplitude, and vibration frequency on bolt loosening, and determined an optimal combination of these three parameters using the orthogonal test method. Zhang et al. [17] observed that bolt self-loosening occurs even when the nut does not rotate owing to fretting wear at the thread surfaces. He proposed that increasing the bolt preload can reduce the relative sliding between the bolt and the nut thread, thereby reducing thread wear and improving the anti-loosening ability of bolted joints. Wang [18] observed that the larger the amplitude of the impact load, the higher the likelihood of bolt loosening. In addition, he determined that for a certain range of initial preloads and friction coefficients between the engagement surfaces of the thread, bolt loosening is less likely to occur. He et al. [19] investigated 
the variation in the bolt preload and the variation in the preload and rotating looseness of the nut under alternating transverse loads. In addition, Hou et al. [20] numerically simulated the loosening behavior of bolts during the early loosening stage and revealed that the preload, load amplitude, and friction coefficient have a significant influence on the degree of reduction in the thread tensioning force during the material loosening period. It can be seen that experts and scholars have made great contributions to the influence law of preload on bolt loosening failure, but the research on the influence law of preload on bolt loosening under composite excitation is still insufficient.

The "VDI 2230-2003" standard [21] and "Mechanical Design Manual" [22, 23] are commonly used to evaluate the fatigue life of bolts, and both standards consider the influence of the preload on bolt fatigue. In addition, as bolts are widely used in engineering applications, finite element analyses and the "VDI 2230-2003" standard are often combined to evaluate bolts. Bolts are usually simplified as BEAM unit elements; the forces and moments on the BEAM unit elements can be extracted from the simulation results, and subsequently, the fatigue strength of the bolts can be conveniently calculated using the "VDI 2230-2003" standard checking program. Shi [24] performed fatigue tests on bolted connection structures using different preloads, and obtained a relationship between preload and bolt fatigue life. He also determined the optimal pre-tightening torque range for high-strength bolts. Zhai [25] found that an appropriate increase in the preload can increase the average stress of the bolt and reduce the stress amplitude. Juoksukangas et al. [26] developed a test device to analyze the influence of preloads and cyclic loading on the fatigue life of a single bolted joint. He observed that the fretting fatigue life decreased with the increase in the preload and stress, and verified the test results using a numerical analysis. Song et al. [27] studied the fatigue performance of bolts considering preloads of $10 \%, 30 \%$, and $50 \%$ of the ultimate strength of the bolt material, and plotted curves for the maximum preload and pre-tightening torque of 8.8 grade M8 and M27 bolts with different stress ratios. Tang et al. [28] revealed that an insufficient initial preload shortens the fatigue life of bolts, and sufficient preloading is required to ensure the fatigue performance of bolted connections. However, an excessive initial preload can cause tensile deformation of the bolt and can affect its usability. Therefore, preloads have a significant influence on the fatigue strength of bolts, and an appropriate preload must be selected based on engineering requirements.

The existing research has made significant contributions to improve the understanding of the influencing factors of bolt loosening and fatigue, failure evaluation methods, simulation methods, preload application methods, and the influence of preloads. However, bolt loosening and fatigue are not independent processes, and a competitive failure relationship exists between them depending on the actions of different loads. Bolts under transverse loads primarily exhibit loosening failure, whereas bolts under axial loads primarily exhibit fatigue failure. Furthermore, transverse loads accelerate bolt fatigue and axial loads affect bolt loosening. However, existing research on the competitive failure relationship and failure mechanism of bolt loosening and fatigue under different preloads is insufficient. Therefore, the competitive failure relationship and the influence of preloads on competitive failure under composite excitation must be studied to better predict the failure mode and failure life of bolted joints.

In this study, we perform competitive failure tests of bolt loosening and fatigue under different preloads, and investigate the competitive relationship between loosening and fatigue failure under composite excitation, as well as the influence of preloads on competitive failure. The competitive failure phenomenon of bolt loosening and fatigue under composite excitation is verified, and the influence parameter that determines the competitive failure mode and failure life of the bolt is determined. Subsequently, the influence of preloads on the competitive failure of bolts is analyzed. Finally, we propose an equation to calculate the optimal preload of 8.8 grade high-strength bolts, which can serve as a reference for engineering applications.

\section{Competitive Failure Test of Bolts under Different Preloads}

Competitive failure tests were performed on bolts under composite excitation using an MTS tensile torsion fatigue testing machine. The axial load on the bolt was directly applied by the testing machine, and the transverse load on the bolt was applied by converting the torque generated by the testing machine to a shear force using a fixture. The test specimens were 8.8 grade $\mathrm{M} 8 \times 1.25 \times 70$ high-strength bolts, and the clamping force was recorded by a pressure sensor. The testing machine and test setup of the specimens are shown in Figure 1. The transverse and axial loads were applied sinusoidally with a phase difference of $90^{\circ}$. The transverse load was controlled by displacement, and the load ratio $\left(\xi_{\mathrm{T}}\right)$ was -1 . The axial load was controlled by force, and the load ratio $\left(\xi_{\mathrm{A}}\right)$ was 0 . For practical engineering applications, the bolt preload is typically calculated using Eq. (1):

$$
F_{\mathrm{k}}=k \sigma_{\mathrm{s}} A_{\mathrm{eff}},
$$

where $k$ is the coefficient of the yield strength, $\sigma_{\mathrm{s}}$ is the yield strength of the bolt material, and $A_{\text {eff }}$ is the effective cross-sectional area of the bolt; $A_{\mathrm{eff}}=\pi d_{\mathrm{e}}^{2} / 4$, where $d_{\mathrm{e}}$ is the effective diameter of the bolt. 


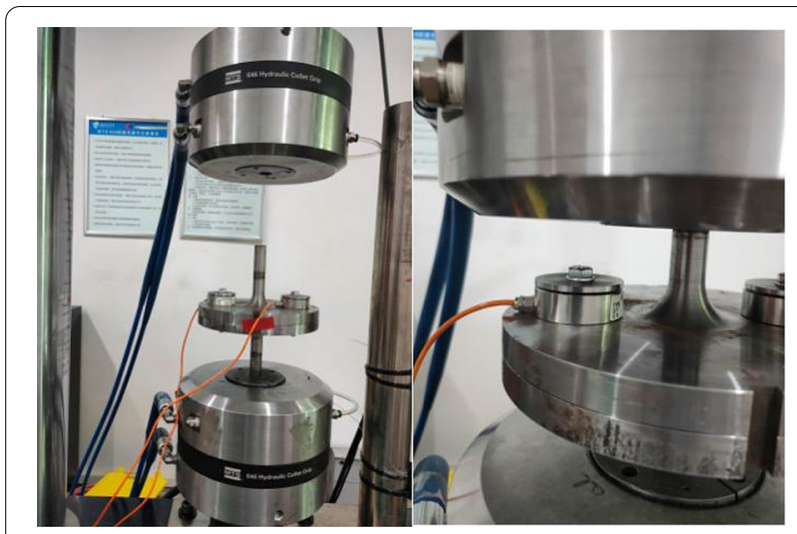

Figure 1 Test setup with fatigue testing machine and specimen

Generally, the initial pre-tightening stress of a bolt should not exceed $80 \%$ of its yield strength [29]. Therefore, in this study, the initial preloads of the bolts were divided into four grades, and the coefficient $k$ of the yield strength was set to $0.3,0.4,0.5$, and 0.6 , respectively. Using Eq. (1), the preloads were calculated as $F_{0.3}=7.03$ $\mathrm{kN}, F_{0.4}=9.37 \mathrm{kN}, F_{0.5}=11.71 \mathrm{kN}$, and $F_{0.6}=14.05 \mathrm{kN}$, respectively. The composite excitation combinations of the bolts with different preloads are shown in Table 1. The fracture modes of the specimens subjected to the competitive failure test are shown in Figure 2. The bolts were either fractured at the first of the bolt working threads or at the transition filet along the bolt head. Fracture failure is most likely to occur at these two positions due to the stress concentration at these positions, and the fact that the composite stress is the highest at these two cross-sections as they are the interfaces at which the bolts contact the clamped parts. In addition, two failure modes were observed for the bolt specimens: loosening failure and fatigue failure, and the fracture mode is not unique. This demonstrates that a competitive relationship exists between the two failure modes depending on the different load forms.

\section{Analysis of Bolt Competitive Failure}

\subsection{Recession Curve of Clamping Force}

The recession curves of the bolt clamping force under composite excitation are continuous and decrease smoothly. In general, the curve can be divided into three stages: the material loosening period, the structural loosening period, and the fatigue fracture period [30]. During the material loosening period, the plastic deformation of the bolt material rapidly reduces the bolt clamping force; during the structural loosening period, the relative movement between the internal and external thread contact surfaces of the bolt causes the nut to rotate, which slowly reduces the bolt clamping force; finally, during the fatigue
Table 1 Composite excitation under different preloads

\begin{tabular}{|c|c|c|c|c|}
\hline Preload & $F_{\mathrm{T}, \mathrm{a}}(\mathrm{mm})$ & $F_{\mathrm{A}, \mathrm{a}}(\mathrm{kN})$ & $R(\mathrm{~mm} / \mathrm{kN})$ & $\begin{array}{l}\text { Number } \\
\text { of valid } \\
\text { specimens }\end{array}$ \\
\hline \multirow[t]{10}{*}{$F_{0.3}$} & 0.5 & 10 & 0.05 & 3 \\
\hline & 0.6 & 0 & - & 4 \\
\hline & 0.6 & 2 & 0.3 & 3 \\
\hline & 0.6 & 3 & 0.2 & 3 \\
\hline & 0.6 & 4 & 0.15 & 4 \\
\hline & 0.6 & 6 & 0.1 & 3 \\
\hline & 0.6 & 8 & 0.075 & 3 \\
\hline & 0.6 & 10 & 0.06 & 3 \\
\hline & 0.8 & 8 & 0.1 & 3 \\
\hline & 0.8 & 10 & 0.08 & 3 \\
\hline \multirow[t]{11}{*}{$F_{0.4}$} & 0.5 & 10 & 0.05 & 2 \\
\hline & 0.6 & 0 & - & 2 \\
\hline & 0.6 & 2 & 0.3 & 3 \\
\hline & 0.6 & 3 & 0.2 & 2 \\
\hline & 0.6 & 4 & 0.15 & 2 \\
\hline & 0.6 & 6 & 0.1 & 2 \\
\hline & 0.6 & 8 & 0.075 & 3 \\
\hline & 0.6 & 10 & 0.06 & 3 \\
\hline & 0.8 & 8 & 0.1 & 2 \\
\hline & 0.8 & 10 & 0.08 & 3 \\
\hline & 1.0 & 10 & 0.1 & 3 \\
\hline \multirow[t]{10}{*}{$F_{0.5}$} & 0.5 & 10 & 0.05 & 3 \\
\hline & 0.6 & 0 & - & 4 \\
\hline & 0.6 & 2 & 0.3 & 4 \\
\hline & 0.6 & 3 & 0.2 & 3 \\
\hline & 0.6 & 4 & 0.15 & 5 \\
\hline & 0.6 & 6 & 0.1 & 3 \\
\hline & 0.6 & 8 & 0.075 & 4 \\
\hline & 0.6 & 10 & 0.06 & 4 \\
\hline & 0.8 & 8 & 0.1 & 3 \\
\hline & 0.8 & 10 & 0.08 & 3 \\
\hline \multirow[t]{9}{*}{$F_{0.6}$} & 0.5 & 8 & 0.0625 & 3 \\
\hline & 0.6 & 0 & - & 2 \\
\hline & 0.6 & 2 & 0.3 & 5 \\
\hline & 0.6 & 4 & 0.15 & 5 \\
\hline & 0.6 & 6 & 0.1 & 3 \\
\hline & 0.6 & 8 & 0.075 & 3 \\
\hline & 0.6 & 10 & 0.06 & 3 \\
\hline & 0.8 & 8 & 0.1 & 3 \\
\hline & 0.8 & 10 & 0.08 & 3 \\
\hline
\end{tabular}

Note: $F_{\mathrm{T}, \mathrm{a}}$ is the transverse displacement amplitude, $F_{\mathrm{A}, \mathrm{a}}$ is the axial load amplitude, and $R$ is the ratio of the transverse load to the axial load, $R=F_{\mathrm{T}, \mathrm{a}} / F_{\mathrm{A}, \mathrm{a}}$

fracture period, the bolt cracks and expands, resulting in an instantaneous fracture of the bolt, and the clamping force reduces to zero. Notably, an explicit and unified dividing method to determine the dividing points of each stage of the recession curve of the bolt clamping force 


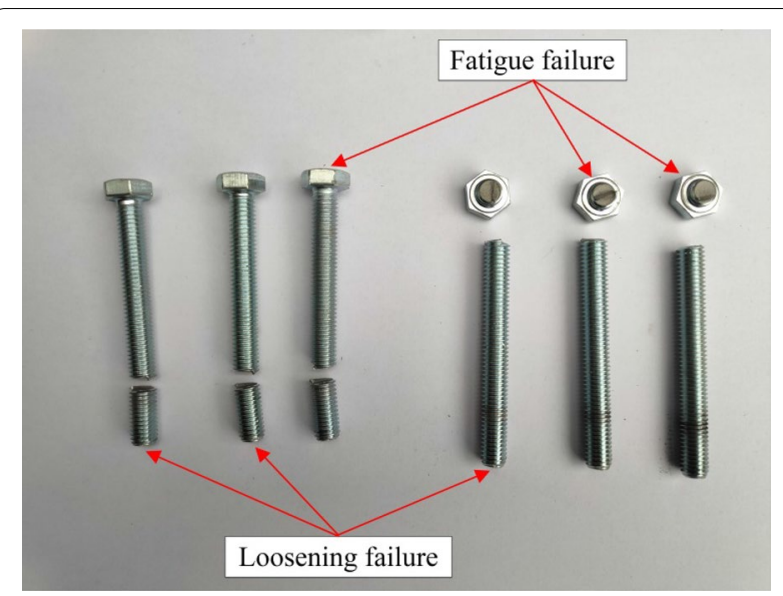

Figure 2 Fracture modes of bolt specimens

has not been established in previous studies, and the determinant condition of bolt loosening and fatigue has also remained undefined.

Since the load applied to the bolt is sinusoidal, the clamping force of the bolt is fluctuating, and the recession curve of bolt clamping force is a load broadband. In order to obtain the dividing point between the structural loosening period and the fatigue fracture period, we propose to use the tangent point between the mean curve of the clamping force broadband and the tangent line with a specific angle as the dividing point. Based on a statistical analysis of the bolt clamping force recession curves obtained herein, we found that when the tangent angle changes from $0^{\circ}$ to $90^{\circ}, 45^{\circ}$ is the best angle, so the tangent point of the the mean curve of the clamping force broadband with a $45^{\circ}$ tangent can serve as the dividing point between the structural loosening period and the fatigue fracture period (denoted by $N_{2 k}, k=0.3,0.4,0.5$, and 0.6). This tangent point is unique. The bolt clearly demonstrates structural loosening before the tangent point, and fatigue fracture after the tangent point, which proves the correctness of the method. Moreover, Jiang et al. [9] believed that for high-strength bolts with hard material, the clamping force was reduced to about $90 \%$ of the initial preload when the bolt entered the structural loosening period, but this definition was conservative. Jiang et al. [31] took the moment when the clamping force was reduced to about $70 \%$ of the initial preload as the standard for full loosening of high-strength bolts, which was dangerous. We find that for high-strength bolts, when the clamping force of the bolt was reduced to $80 \%$ of the initial preload, the bolt fully entered the structural loosening period; therefore, the clamping force can be used to determine whether there is significant bolt loosening. If the bolt clamping force reduces to $80 \%$ of the initial preload during the structural loosening period (denoted by $N_{\mathrm{L} k}$ ), i.e., $N_{\mathrm{L} k}<N_{2 k}$, the bolt undergoes loosening failure; if the bolt clamping force does not reduce to $80 \%$ of the initial preload until the fatigue fracture period (denoted by $N_{\mathrm{F}_{-} k}$ ), i.e., $N_{\mathrm{F}_{-} k}>N_{2 \_k}$, the bolt undergoes fatigue failure.

The recession curves of the bolt clamping force with $R=0.15 \mathrm{~mm} / \mathrm{kN}$ and $R=0.06 \mathrm{~mm} / \mathrm{kN}$ were obtained as shown in Figure 3 (0.6-4 means that the transverse displacement amplitude is $0.6 \mathrm{~mm}$ and the axial load amplitude is $4 \mathrm{kN}$, and $0.6-10$ is also defined in the same way). As shown, under different initial preloads, when $R=0.15$ $\mathrm{mm} / \mathrm{kN}$, all the recession curves satisfy the condition $N_{\mathrm{L}_{-} k}<N_{2-k}$, i.e., the bolts undergo loosening failure; when $R=0.06 \mathrm{~mm} / \mathrm{kN}$, all the recession curves satisfy the condition $N_{\mathrm{F}_{-} k}>N_{2 \_k}$ i.e., the bolts undergo fatigue failure.
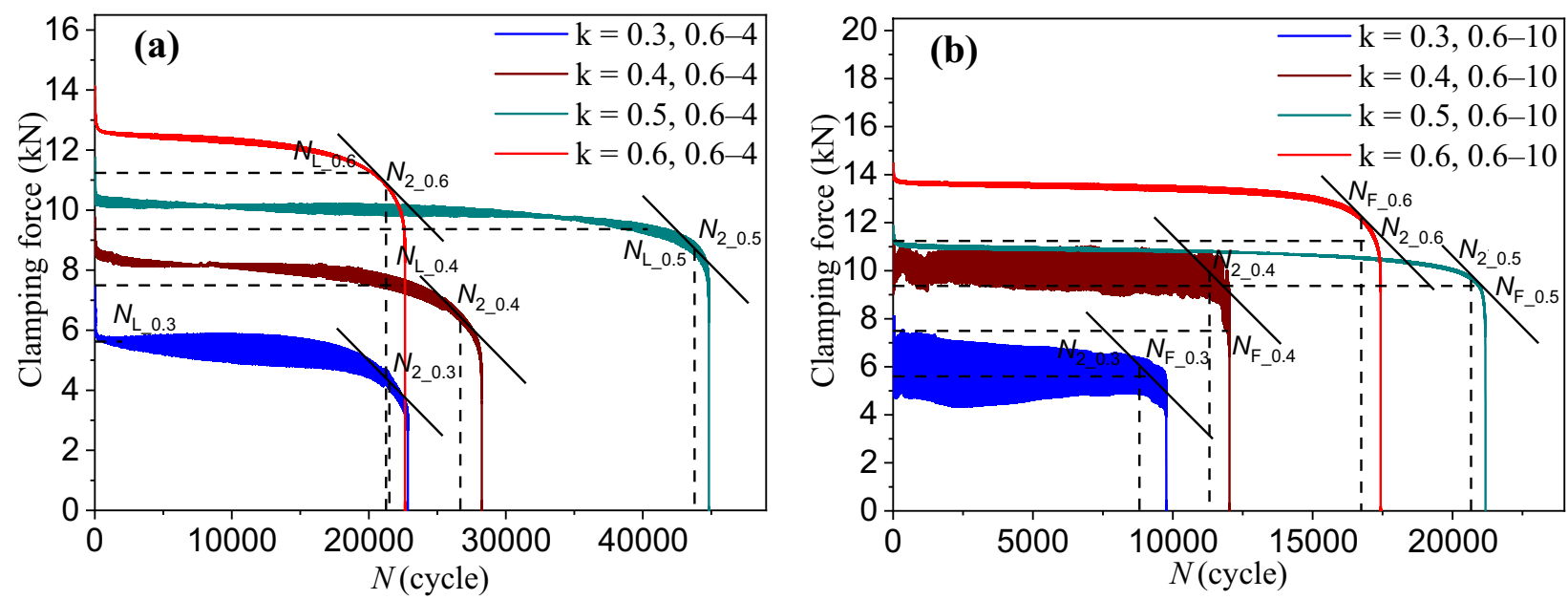

Figure 3 Recession curves of bolt clamping force under different preloads: $\mathbf{a} R=0.15 \mathrm{~mm} / \mathrm{kN}, \mathbf{b} R=0.06 \mathrm{~mm} / \mathrm{kN}$ 
Furthermore, the failure life of the bolt first increases and then decreases with the increase in the initial preload at the same $R$, and the failure life of the bolt differs with the same transverse load but different axial loads. Therefore, the failure life of a bolt is determined by the load amplitude and the preload, and the optimal preload of 8.8 grade M8 $\times 1.25 \times 70$ high-strength bolts for the longest life is $F_{0.5}$.

\subsection{Influence Law of Load Ratio}

To further study the influence of $R$ on the competitive bolt failure mode according to the criterion established in Figure 3, the failure modes of the of all the bolt specimens listed in Table 1 were distinguished, and the failure mode of each bolt specimen was obtained. To intuitively visualize the failure modes of the bolts, the $R$-significant degree of failure curves under different preloads were plotted, as shown in Figure 4(a) (as the bolts undergo loosening failure when $R>0.15 \mathrm{~mm} / \mathrm{kN}$, they are not shown in the figure); $S_{\mathrm{L}}$ represents the significant degree of bolt loosening failure and $S_{\mathrm{F}}$ represents the significant degree of bolt fatigue failure. $S_{\mathrm{L}}$ and $S_{\mathrm{F}}$ were calculated using the following equations:

$$
\begin{aligned}
& S_{\mathrm{L}}=100 \% \frac{N_{2_{k}}-N_{\mathrm{L}_{k}}}{N_{2_{k}}}, \\
& S_{\mathrm{F}}=100 \% \frac{N_{2_{k}}-N_{\mathrm{F}_{k}}}{N_{2_{k}}} .
\end{aligned}
$$

Based on Figure 4(a), it can be concluded that when $R \leq$ $0.06 \mathrm{~mm} / \mathrm{kN}$, the bolts undergo fatigue failure, and when $R \geq 0.08 \mathrm{~mm} / \mathrm{kN}$, the bolts undergo loosening failure.
Therefore, there is a clear competitive failure relationship between the two failure modes under composite excitation, and a critical value of $R$ exists for bolt loosening failure and fatigue failure. When the ratio of the transverse load to the axial load is higher than the critical value of $R$, bolt loosening failure occurs, and when it is less than the critical value of $R$, bolt fatigue failure occurs. This implies that the critical $R$ value is an inherent property of the bolt that is related to the bolt material, size, and assembly method, but is unrelated to the magnitude of the load. In addition, due to the large preload when $k=0.6$, the bolt is prone to local plastic deformation, which makes the data dispersion of loosening failure relatively large and makes the curve fluctuate significantly. Furthermore, Figure 4(a) shows that when the numerical value of $R$ is around 0.075 $\mathrm{mm} / \mathrm{kN}$, the bolt reaches a critical state of loosening and fatigue, and $S_{\mathrm{L}}$ and $S_{\mathrm{F}}$ tend toward zero. Therefore, the critical $R$ value of loosening and fatigue failure of 8.8 grade M8 $\times 1.25 \times 70$ high-strength bolts under composite excitation is $0.075 \mathrm{~mm} / \mathrm{kN}$. In addition, the $R$-significant degree of failure curves under different preloads have the same failure regularity, which proves that the failure mode of the bolt is only determined by $R$ and is unaffected by the initial preload. Thus, the failure mode of bolts subjected to composite excitation can be directly predicted based on the critical $R$ value.

The $R$-life curves under different preloads are shown in Figure 4(b). As shown, the failure life of the bolt first increases significantly, and then gradually decreases with the increase in $R$ under composite excitation (except when the axial load is zero). When the transverse load is constant, the axial load gradually decreases with the increase in $R$. Conversely, as the axial load increases, the failure life of the bolt first increases slowly and then

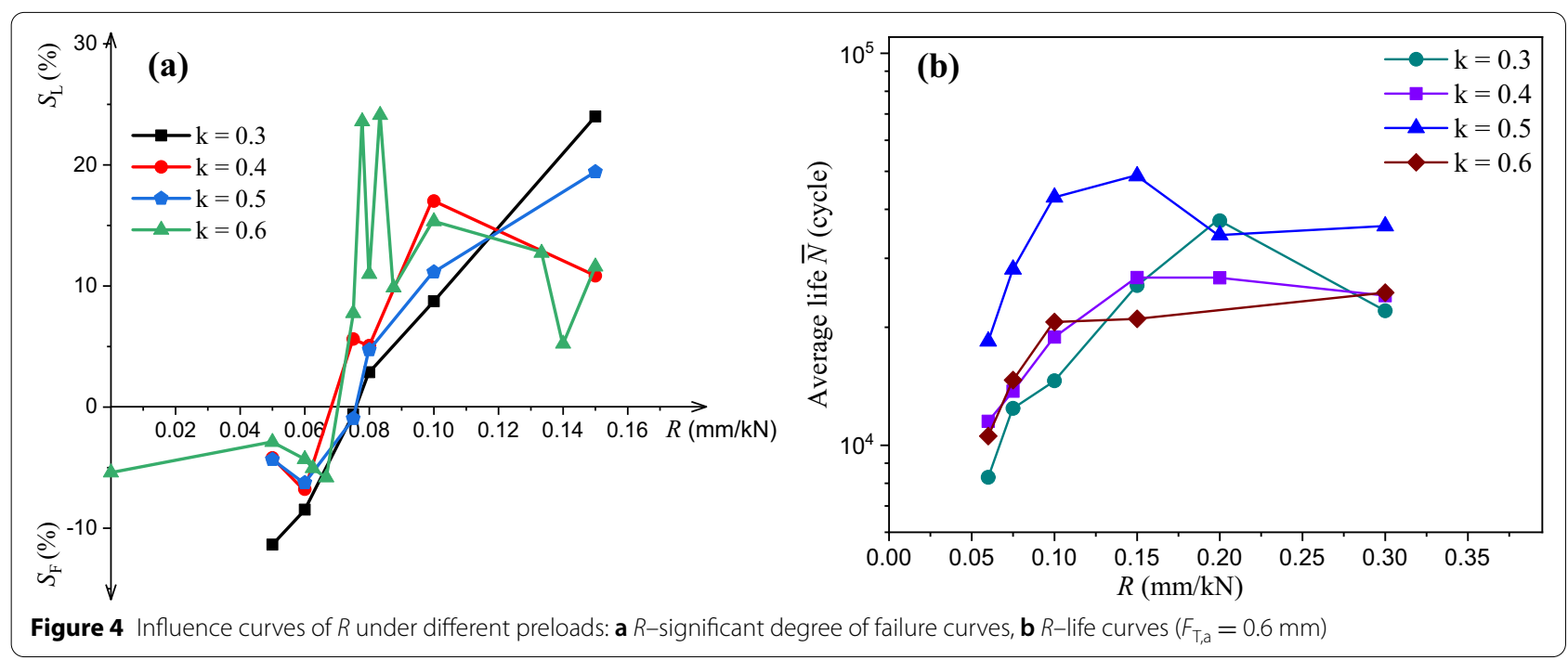


decreases rapidly. This indicates that a small axial load restrains bolt failure; when the axial load exceeds a certain value, the large composite stress of the bolt under composite excitation causes a large plastic deformation in the thread, which reduces the fatigue life of the bolt. The force diagrams of the bolt thread surface under composite excitation are shown in Figure $5\left(F_{\mathrm{k}}\right.$ is the initial preload of the bolt, $F_{\mathrm{T}}$ is the external transverse excitation, $F_{\mathrm{SA}}$ is the axial excitation of the bolt, $\alpha$ is the helix angle, $\mu_{1}$ is the static friction coefficient, and $\mu_{2}$ is the sliding friction coefficient). According to Junker's oblique block model [3], when the bolt is not subjected to an external load, although the block has a tendency to slide downward, the inclined plane and the block remain in a static state, i.e., the bolt thread is in a self-locking state, because the downward force $F_{\mathrm{k}} \sin \alpha$ along the inclined plane is less than the static friction force $\mu_{1} F_{k} \cos \alpha$. When the bolt is only subjected to an external transverse excitation $F_{\mathrm{T}}$, relative sliding motion occurs between the inclined plane and the block, and the friction between them changes from static friction to sliding friction. As the sliding friction force $\mu_{2} F_{\mathrm{k}} \cos \alpha$ is less than $\mu_{1} F_{\mathrm{k}} \cos \alpha$, the self-locking state of the thread is broken and the block begins to slide downward, i.e., the internal and external threads of the bolt move relative to each other, and the bolt becomes loose. When the bolt is affected by composite excitation, the axial load of the bolt increases and becomes the resultant force of $F_{\mathrm{k}}$ and $F_{\mathrm{SA}}$, the downward force along the inclined plane becomes $\left(F_{\mathrm{k}}+F_{\mathrm{SA}}\right)$ $\sin \alpha$, and the static friction force becomes $\mu_{1}\left(F_{\mathrm{k}}+F_{\mathrm{SA}}\right)$ $\cos \alpha$. The transverse excitation $F_{\mathrm{T}}$ leads to transverse sliding between the thread contact surfaces; however, owing to the increase in the positive pressure on the inclined plane and the block contact surfaces, the sliding friction force $\mu_{2}\left(F_{\mathrm{k}}+F_{\mathrm{SA}}\right) \cos \alpha$ is higher than $\left(F_{\mathrm{k}}+F_{\mathrm{SA}}\right) \sin \alpha$ within a certain range. Consequently, no relative movement occurs between the thread contact surfaces, and the bolt does not become loose. Therefore, to a certain extent, small axial loads can restrain bolt failure; however, when the axial load increases, the large composite tensile and shear stress causes a large plastic deformation in the thread surfaces, and the large axial load accelerates bolt failure, thereby reducing the fatigue life of the bolt.

\subsection{Load-life Curves}

The load-life curves of the bolts subjected to composite excitation were plotted as shown in Figure 6(a) (the ordinate is a logarithmic coordinate). As shown, when the transverse load is constant, the axial load-life curves do not decrease monotonously. At small axial loads, the failure life of some bolts is longer than that under pure transverse loads. Therefore, axial loads do not accelerate the failure of the bolt. The above analyses prove that small axial loads can restrain bolt failure. As shown in Figure 6(b), at different initial preloads, the significant degree of the restraining effect of small axial loads on bolt failure varies. When the preloads are small $(k=0.3$, $0.4,0.5)$, except for some singular data points, the axial loads generally accelerate the bolt failure, but when the preloads are large $(k=0.6)$, the small axial loads restrain the bolt failure. This is because under composite excitation, the downward force along the thread is $\left(F_{\mathrm{k}}+F_{\mathrm{SA}}\right)$ $\sin \alpha$, the transverse static friction force is $\mu_{1}\left(F_{\mathrm{k}}+F_{\mathrm{A}}\right) \cos \alpha$, and the transverse sliding friction force is $\mu_{2}\left(F_{\mathrm{k}}+F_{\mathrm{A}}\right) \cos \alpha$. When $F_{\mathrm{k}}$ decreases, the positive pressure on the thread and the transverse static friction force decrease. If $F_{\mathrm{T}}$ is higher than $\mu_{2}\left(F_{\mathrm{k}}+F_{\mathrm{A}}\right) \cos \alpha$, transverse relative movement occurs between the thread surfaces, and the upward friction along the thread surface changes from $\mu_{1}\left(F_{\mathrm{k}}+F_{\mathrm{A}}\right)$ $\cos \alpha$ to $\mu_{2}\left(F_{\mathrm{k}}+F_{\mathrm{A}}\right) \cos \alpha$. As $\mu_{2}\left(F_{\mathrm{k}}+F_{\mathrm{A}}\right) \cos \alpha$ cannot offset the downward force $\left(F_{\mathrm{k}}+F_{\mathrm{SA}}\right) \sin \alpha$ along the thread surface, the bolt becomes loose and the failure process of the bolt accelerates. Thus, when the preload is large, the axial load can restrain the bolt failure to some extent; when the preload is small, the clamping load tends to zero, the thread contact surfaces experience a downward relative movement, and the axial load can no longer restrain the bolt failure. In addition, Figure 6(a) also indicates that the average failure life of a bolt is affected by both the external load and the initial preload, and the load-life curve

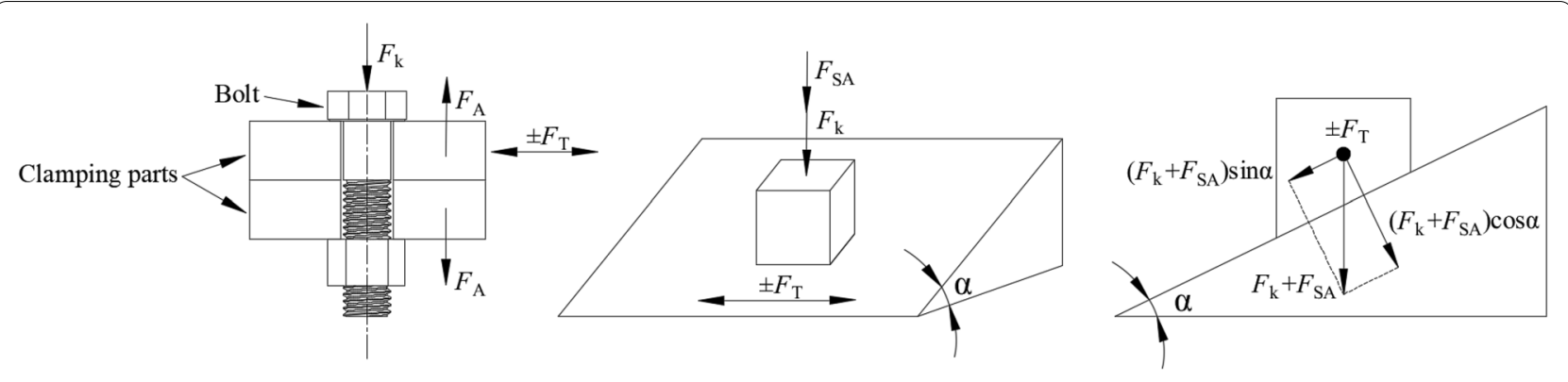

Figure 5 Schematic diagram of force on thread surface 

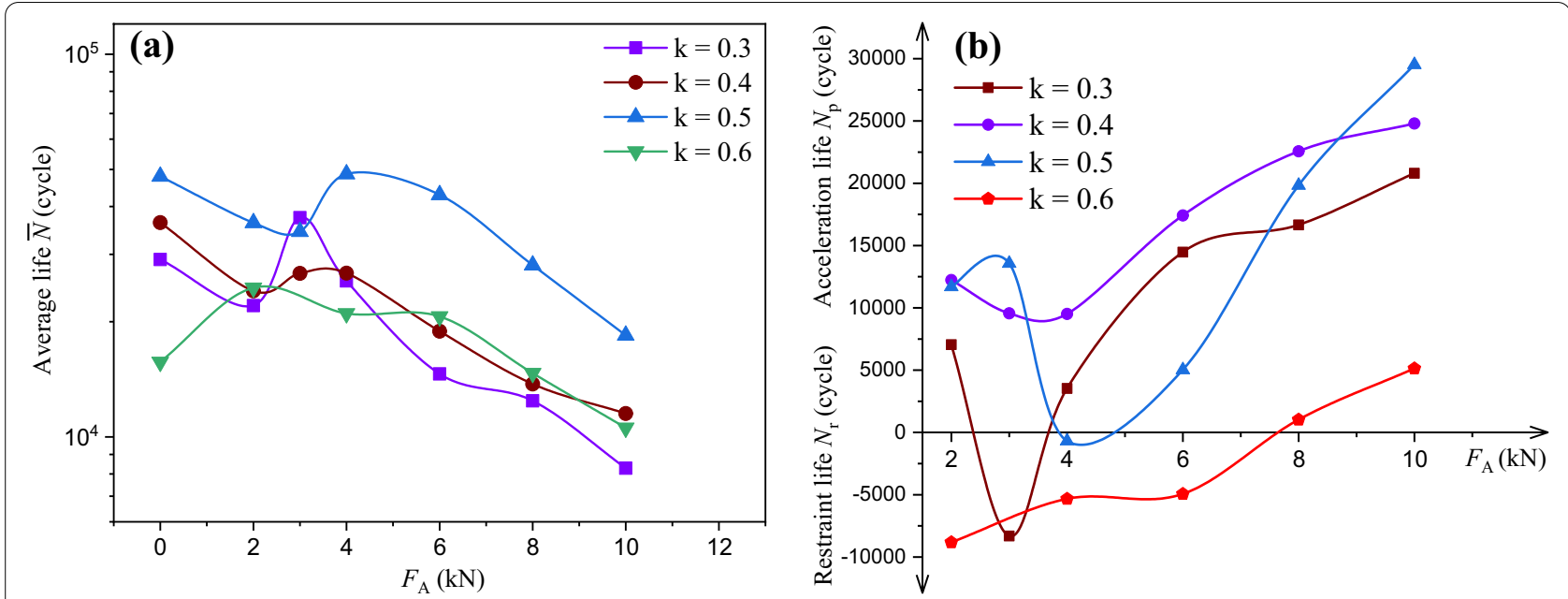

Figure 6 Load-life curves $\left(F_{T, a}=0.6 \mathrm{~mm}\right)$ : a load-life curves, $\mathbf{b}$ significant degree of axial load restraining or accelerating bolt failure

with $k=0.5$ is the highest. This further proves that the failure life of a bolt is determined by the load amplitude and the preload, and the optimal preload for 8.8 grade $\mathrm{M} 8 \times 1.25 \times 70$ high-strength bolts is $F_{0.5}$.

\section{Fracture Analysis}

The crack propagation law of bolt fracture under composite excitation and the influence of the initial preload on cracks were analyzed herein. A JSM-6610LV scanning electron microscope was used to observe the fracture surfaces of bolts fractured under composite excitation $(0.6 \mathrm{~mm}-4 \mathrm{kN})$ with different preloads. The macroscopic fracture morphology is shown in Figure 7. The typical fatigue fractures can be divided into three areas based on their morphological characteristics, namely, the crack source area, the crack propagation area, and the instantaneous fracture area [32]. During the crack initiation stage, the shear stress plays a leading role in crack formation, and cracks form along the maximum shear strain plane. Once the cracks form, the normal stress begins to dominate, and the cracks expand along the depth and sides under the cyclic action of the normal stress. As shown in Figure 7, the bolt fracture also has a crack source area (a), a crack propagation area (b), and an instantaneous fracture area (c). From a macroscopic viewpoint, the composite stress at the two contact interfaces between the bolt and clamped parts is the highest; the stress concentration leads to fatigue damage accumulation at these two interfaces, which eventually causes bolt fracture. From a microscopic viewpoint, the bolt crack source is generated due to shear stress, whereas the normal stress increases the crack expansion, leading to bolt fracture. As the transverse load of the composite excitation generates shear stress and the stress ratio is -1 , the crack source is symmetrically distributed on both sides of the bolt fracture. Multiple cracks are initiated on the circumferential surface of the bolt that propagate radially to the interior, forming a typical multi-crack initiation failure. The axial load generates normal stress and the stress ratio is zero; consequently, the bolt is always in a tensile state. Therefore, once a crack has formed, it steadily expands along the depth and sides under the cyclic action of the normal stress. Eventually, it breaks instantaneously in the middle of the fracture and produces an instantaneous fracture area. The crack propagation area is located between the crack source area and the instantaneous fracture area.

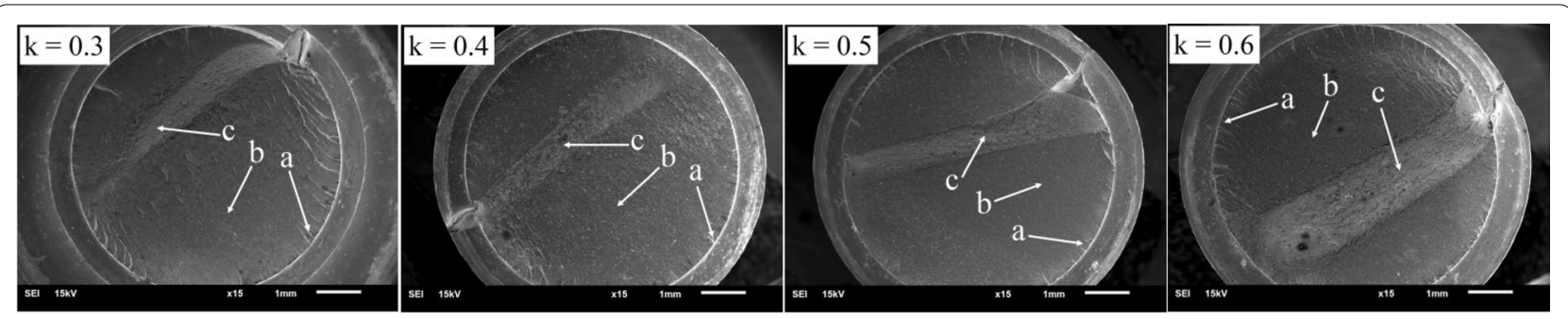

Figure 7 Macro morphology of bolt fracture 
Owing to the different initial preloads of the bolts, the three fracture areas also have several differences. With large preloads, the crack propagation speeds on both sides are similar. The instantaneous fracture area is closer to the center of the bolt fracture, the area is wide, and the fracture step is not pronounced. As the preload decreases, there is a significant difference in the crack propagation speed on either side. The instantaneous fracture area gradually moves to one side and narrows, and the fracture step becomes pronounced. This is because under transverse loads, multiple cracks occur on both sides of the circumferential surface of the bolt and propagate along the depth and sides owing to the axial load. Large preloads can offset the external axial loads, ensuring that the clamping force is always greater than zero, the bolt always experiences normal stress, and the stress is symmetrically distributed on both sides of the fracture. Therefore, the crack propagation speed on either side of the fracture is essentially equal, and the instantaneous fracture area is close to the center of the bolt fracture. However, small preloads cannot offset the axial load, and the clamping force can eventually become zero. When the normal stress varies between zero and a positive value, the crack propagation speed on one side becomes faster than that on the other side due to the thread helix angle, leading to a gradual change in the tensile area of the bolt and the eccentricity of the axial load. Therefore, the crack propagation speed on the side with the faster propagation is further accelerated until fracture. Owing to the superposition of the eccentric load and helix angle, the step of the bolt fracture becomes more pronounced, and the instantaneous fracture area becomes inclined towards one side when the preload is small. In addition, as a larger preload contributes to a larger axial load, when the crack propagates to a shallow depth, the remaining adhesion area breaks due to the higher normal stress. Conversely, when the preload is smaller, the normal stress is lower, and the broken adhesion area is also correspondingly smaller. Thus, as the preload increases, the width of the instantaneous fracture area also increases.

The crack source area (a), crack propagation area (b), and instantaneous fracture area (c) of the bolt fracture surface were further enlarged, and their micromorphology was observed, as shown in Figure 8. As shown in Figure 8(a), several radial fiber stripes in the crack propagation area gather in the crack source area, forming multiple radial propagation cracks. Consequently, the fracture is a typical multi-crack initiation failure.

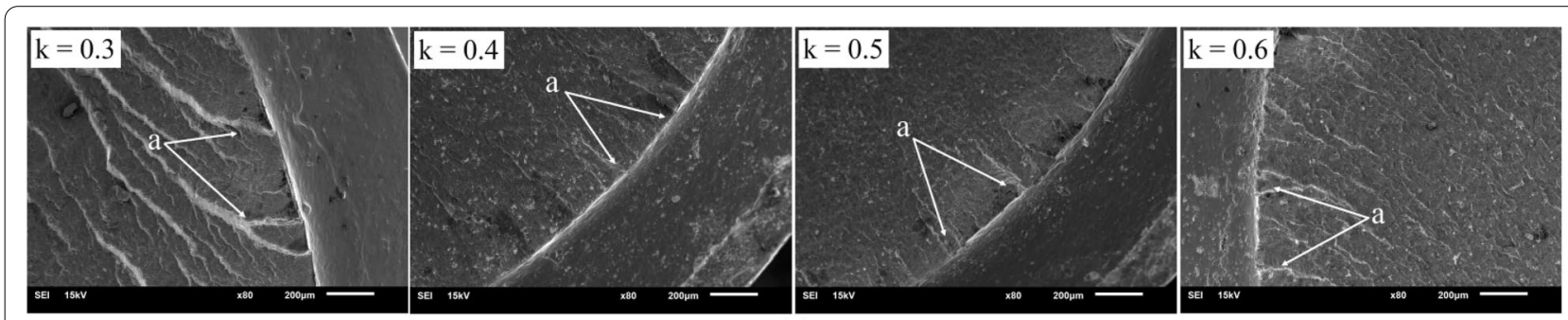

(a)
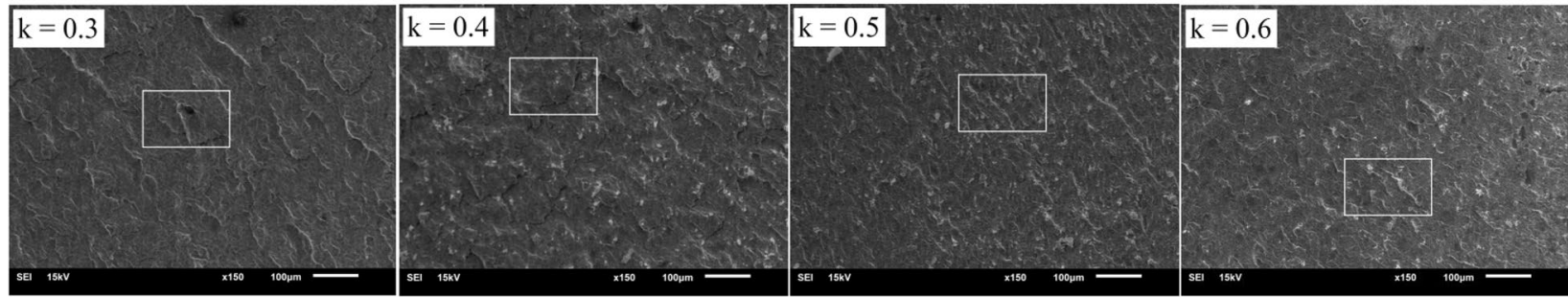

(b)
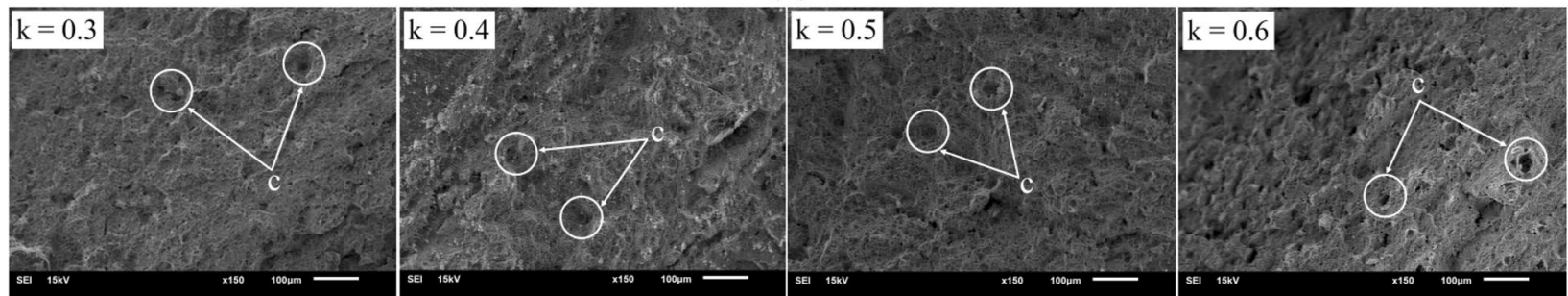

(c)

Figure 8 Microscopic morphology of bolt fracture: a crack source area, b crack propagation area, c instantaneous fracture area 
With small preloads, the crack source area is relatively flat and bright, the fracture surface is significantly worn, the ridge morphology is not pronounced, and the surface has a porcelain-like structure. This is because when the preload is small, the resultant forces of $F_{\mathrm{k}}$ and $F_{\mathrm{SA}}$ on the bolt are small, the normal stress is low, and the shear stress generated by the transverse load $F_{\mathrm{T}}$ dominates. Due to the shear stress, the fracture is repeatedly scratched and worn, and the crack source area becomes relatively smooth and has a porcelain-like structure. As the preload increases, the normal stress also increases, which restrains the scratch and wear effect of the shear stress. Consequently, the crack source area becomes rough and a ridge morphology gradually appears. The crack propagation areas are shown in Figure 8(b). As shown, the smoothness and flatness of the crack propagation areas vary with the preload. When the preload is large, the normal stress is high, which restrains the friction and wear effect of the shear stress. Consequently, the crack propagation area is rough, and the fatigue striations and white ligaments are finer and clearer. In contrast, when the preload is small, the crack propagation area is characterized by smooth flakes owing to the abrasion and wear effect of the shear stress. The instantaneous fracture area is shown in Figure 8(c). As shown, a large number of loose dimples are present. When the preload is large, the normal stress restrains the friction and wear effect of the shear stress, and the dimples in the instantaneous fracture area become larger, deeper, and irregular, forming typical equiaxed dimples. When the preload is small, these dimples become smaller, shallower, and more regular owing to the friction and wear effect of the shear stress, forming typical shear dimples. In summary, the microstructure of the bolt fracture reveals that bolt fracture is affected by composite excitation and preload. Owing to the different dominant effects of the normal stress and shear stress, the macroscopic fracture morphology differs significantly, which further proves that a competitive relationship exists between bolt loosening failure and fatigue failure, and that the axial load has restraining effect on bolt failure to some extent.

\section{Conclusions}

(1) An obvious competitive failure relationship exists between bolt loosening and fatigue under composite excitation. The bolt failure mode under different preloads is determined by the ratio of the transverse load to the axial load of the composite excitation, and is unrelated to the magnitude of the preload and the excitation amplitude. The failure mode can be predicted based on the critical $R$ value.
(2) The failure life of a bolt is determined by the preload, and the excitation amplitude and failure life of the bolt can be predicted based on the magnitude of the load. For 8.8 grade high-strength bolts, the optimal preload can be calculated as $F_{\mathrm{k}}=0.5 \sigma_{\mathrm{s}} A_{\text {eff }}$.

(3) Under composite excitation, small axial loads can restrain bolt failure depending on the preload. When the preload is large, the axial load can restrain bolt failure to a certain extent, whereas when the preload is small, the axial load cannot restrain bolt failure.

(4) When the preload is small, bolt fracture is primarily affected by the friction and wear caused by shear stress. When the preload is large, bolt fracture is primarily affected by normal stress, which restrains the friction and wear effect of the shear stress, which proves that a competitive failure relationship exists between bolt loosening and bolt fatigue, and that axial loads can restrain bolt failure.

\section{Acknowledgements}

Not applicable.

\section{Authors' contributions}

LY and GY were in charge of the whole trial; LY wrote the manuscript; SX, SJ and JC assisted with sampling and laboratory analyses. All authors read and approved the final manuscript.

\section{Authors' Information}

Guangwu Yang, born in 1977, is currently a professor at State Key Laboratory of Traction Power, Southwest Jiaotong University, China. He received his PhD degree on Vehicle Engineering from Southwest Jiaotong University, China, in 2005. His main research interests include vehicle structure strength and fatigue.

Long Yang, born in 1993, is currently a PhD candidate at State Key Laboratory of Traction Power, Southwest Jiaotong University, China. He received his bachelor degree from Lanzhou Jiaotong University, China, in 2017. His research interests include failure mechanism of bolt fasteners.

Jingsong Chen, born in 1997, is currently a master candidate at State Key Laboratory of Traction Power, Southwest Jiaotong University, China. He received his bachelor degree from Dalian Jiaotong University, China, in 2020. His research interests include vehicle structure strength and fatigue life prediction. Shoune Xiao, born in 1964, is currently a professor at State Key Laboratory of Traction Power, Southwest Jiaotong University, China. He received his master degree on Solid Mechanics from Southwest Jiaotong University, China, in 1988. His main research interests include vehicle structure strength and structural optimization.

Shilin Jiang, born in 1995, is currently an engineer at China Railway Engineering Service Co. Ltd, China. He received his master degree from Southwest Jiaotong University, China, in 2020. His research interests include vehicle structure strength and fatigue life prediction.

\section{Funding}

Supported by National Natural Science Foundation of China (Grant No. 51675446), and Independent Subject of State Key Laboratory of Traction Power (Grant No. 2019TPL-T13).

\section{Competing Interests}

The authors declare no competing financial interests.

Received: 21 March 2021 Revised: 13 October 2021 Accepted: 26 Novem- 
ber 2021

Published online: 19 December 2021

\section{References}

[1] Y Chen. Study on the failure mechanism and the effect on mechanical behavior of the whole structure of bolt loosening. Dalian: Dalian University of Technology, 2019. (in Chinese)

[2] ZW Wei. Research on loose mechanism and vibration test method of subway bolts. Hangzhou: Zhejiang Sci-Tech University, 2019. (in Chinese)

[3] G H Junker. New criteria for self-loosening of fasteners under vibration. SAE Transactions, 1969, 78: 314-335.

[4] JY Yang, C Ling, P Wu, et al. Anlysis of fracture reason of high strength bolt. Hot Working Technology, 2019, 48(20): 173-176. (in Chinese)

[5] J Mu. Experimental reasearch on fatigue failure of connecting nodes with high-strength bolts. Chongqing: Chongqing Jiaotong University, 2010. (in Chinese)

[6] YCXie. Analysis of the causes of loosen bolts and measurements to locking assembly. Equipment Manufacturing Technology, 2015, (04): 173-175. (in Chinese)

[7] Y Li, C L Chen, S J Wang, et al. Fatigue life analysis and design improvement of high strength bolts. Journal of Chengdu University (Natural Science Edition), 2018, 37(04): 407-411. (in Chinese)

[8] C H Wang, W L Wang, Q Y Lin, et al. Study on bolt loosening mechanism in vibration condition based on precise model. Astronautical Systems Engineering Technology, 2018, 2(04): 43-51. (in Chinese)

[9] Y Y Jiang, M Zhang, TW Park, et al. An experimental study of self-loosening of bolted joints. Journal of Mechanical Design, 2004, 126(5): 925-931.

[10] X Ke, G W Yang, T Zhu, et al. Study on the pretensioning simulation of bolt by rotation angle method. Machinery Design \& Manufacture, 2020, (11): 125-129. (in Chinese)

[11] C H Wang. Analysis and experimental study on loosening characteristics of bolted connections. Dalian: Dalian University of Technology, 2018. (in Chinese)

[12] F Lu. Loosening mechanism and experimental study of bolted structure under vibration. Nanjing: Southeast University, 2017. (in Chinese)

[13] H Gong, J H Liu, XY Ding. Study on the critical loosening condition toward a new design guideline for bolted joints. ARCHIVE Proceedings of the Institution of Mechanical Engineers Part C Journal of Mechanical Engineering Science, 2018, 233(9): 3302-3316.

[14] Q Zheng, Y C Guo, Y P Wei, et al. Loosening of steel threaded connection subjected to axial compressive impact loading. International Journal of Impact Engineering, 2020, 144: 103662.

[15] J A Sanclemente, D P Hess. Parametric study of threaded fastener loosening due to cyclic transverse loads. Engineering Failure Analysis, 2007, 14(1): 239-249.

[16] Y B Zhang. Experimental study on looseness of bolt connection structure under transverse vibration condition. Urumqi: Xinjiang University, 2018. (in Chinese)

[17] MY Zhang, L T Lu, W J Wang, et al. The roles of thread wear on selfloosening behavior of bolted joints under transverse cyclic loading. Wear, 2017, 394: 30-39.

[18] K P Wang. Research on factors affecting bolt loosening under impact load. Shenyang: Shenyang University of Technology, 2019. (in Chinese)

[19] J F He, T Tang, C S Wang. Finite element research on self-loosening problem of bolt connection under the alternating lateral load. Modern Manufacturing Engineering, 2016, (03): 1-6. (in Chinese)

[20] SY Hou, R D Liao. Influence of ratcheting on self-loosening of bolted joints. Transactions of Beijing Institute of Technology, 2015, 35(09): 924-930. (in Chinese)

[21] VDI 2230 Part 1. Systematic calculation of high duty bolted joints, joints with one cylindrical bolt. Verein Deutscher Ingenieure, 2003.

[22] J Zheng, Y Xu. Mechanical design. Beijing: Peking University Press, 2016. (in (hinese)

[23] D X Cheng. Handbook of mechanical design. 5th ed. Beijing: Chemical Industry Press, 2010. (in Chinese)

[24] H Shi. Research of high strength bolt failure caused by several factors. Shenyang: Northeastern University, 2012. (in Chinese)
[25] X K Zhai. Discussion and research of the calculation method for the fatigue life of the bolt. Manufacturing Automation, 2016, 38(01): 89-91. (in Chinese)

[26] J Juoksukangas, A Lehtovaara, A Mantyla, et al. Experimental and numerical investigation of fretting fatigue behavior in bolted joints. Tribology International, 2016, 103: 440-448

[27] Z M Song, R Li, M Qian, et al. Optimizing prestress of fatigue propertydominated 8.8-grade bolts. Chinese Journal of Materials Research, 2019, 33(08): 629-634. (in Chinese)

[28] C Q Tang, K F Zhou, Y M Mo, et al. FEA Fatigue simulation on the bolt performance. Digital Manufacture Science, 2017, 15(Z1): 30-35.

[29] Z Z Wu, L M Wu. Mechanical design. Beijing: China Railway Publishing House, 2016. (in Chinese)

[30] J H Yu. Feature Analysis and extraction of the loosening of bolted joints with transverse vibration. Beijing: China Academy of Machinery Science and Technology, 2016. (in Chinese)

[31] S L Jiang, G W Yang, S N Xiao, et al. Experimental study on the loosening life of bolts. Journal of Mechanical Strength, 2019, 41(05): 1060-1065. (in Chinese)

[32] Y X Cui, C L Wang. Metal fracture analysis. Harbin: Harbin Institute of Technology Press, 1998. (in Chinese)

\section{Submit your manuscript to a SpringerOpen ${ }^{\circ}$ journal and benefit from:}

- Convenient online submission

- Rigorous peer review

- Open access: articles freely available online

- High visibility within the field

- Retaining the copyright to your article

Submit your next manuscript at $\boldsymbol{\nabla}$ springeropen.com 